\title{
Moufang sets related to polarities in exceptional Moufang quadrangles of type $\mathrm{F}_{4}$
}

\author{
Koen Struyve*
}

\begin{abstract}
Departing from a Moufang set related to a polarity in an exceptional Moufang quadrangle of type $\mathrm{F}_{4}$, we construct a rank three geometry. The main property of this new geometry is that its automorphism group is identical to the one of the underlying Moufang set, providing a tool to study this Moufang set in a geometrical way. As a corollary we obtain that every automorphism of an exceptional Moufang quadrangle of type $F_{4}$ stabilizing the absolute points of a polarity, also centralizes the polarity. This handles the final case of a similar result for all polarities of Moufang $n$-gons with $n \geq 3$.
\end{abstract}

Keywords: Moufang sets, exceptional quadrangles of type $F_{4}$, polarities

MSC 2000: 51E05, 51E12, 20B22

\section{Introduction}

Moufang sets are rank 1 buildings satisfying the Moufang property. They are the glue in constructions of buildings with a big automorphism group (see e.g. [5]) and they play a fundamental role in the classification of certain twin buildings (see [2]). The study of these groups is made harder by the lack of a suitable canonical geometry. In one of his last lectures at Collège de France, Jacques Tits suggested to remedy this by defining a kind of 2-design using the nilpotent structure of the root groups. A general problem is then to determine the collineation group of this geometry with the hope to obtain a Fundamental Theorem for the Moufang sets, i.e., the canonical situation should be that the automorphism group of the geometry is precisely the automorphism group of the Moufang

\footnotetext{
*The author is supported by the Fund for Scientific Research - Flanders (FWO - Vlaanderen)
} 



$$
\begin{aligned}
& {\left[(x, y, b)_{1},(u, v, a)_{4}\right]=(b u+\alpha(}\bar{x} v+\beta y \bar{v}), b v+x u+\beta y \bar{u} \\
& b^{2} a+a \alpha\left(x \bar{x}+\beta^{2} y \bar{y}\right) \\
&\left.+\alpha\left(u^{2} x \bar{y}+\bar{u}^{2} \bar{x} y+\alpha\left(\bar{v}^{2} x y+v^{2} \bar{x} \bar{y}\right)\right)\right)_{2} \\
& \cdot\left(a x+\bar{u}^{2} y+\alpha v^{2} \bar{y}, a y+\beta^{-2}\left(u^{2} x+\alpha v^{2} \bar{x}\right)\right. \\
& a b+b \beta^{-1}(u \bar{u}+\alpha v \bar{v}) \\
&\left.+\alpha\left(\beta^{-1}(x u \bar{v}+\bar{x} \bar{u} v)+y \bar{u} \bar{v}+\bar{y} u v\right)\right)_{3} .
\end{aligned}
$$

These Moufang quadrangles were discovered by Richard Weiss in 1997 in preparation of [6]. In 1999 Bernhard Mühlherr and Hendrik Van Maldeghem gave an algebraic interpretation to these quadrangles by constructing them out of an involution of an $\mathrm{F}_{4}$ Moufang building [3], furthermore they showed in [4] that these quadrangles have polarities if and only if there is a Tits endomorphism $\phi$ of $\mathbb{L}$ (i.e., an endomorphism such that $\left(x^{\phi}\right)^{\phi}=x^{2}$ for all $x$ in $\mathbb{L}$ ) with image $\mathbb{L}^{\prime}$ such that the restriction of $\phi$ to $\mathbb{K}$ is also a Tits endomorphism with image $\mathbb{K}^{\prime}$. In this case $\beta^{\phi}$ will be equal to $\alpha$. We let $\theta$ be the inverse of $\phi$ (defined on $\mathbb{L}^{\prime}$ ). From now on, we will always suppose we have a polarity and we have chosen our coordinate system in such a way that this polarity takes the easy form:

$$
\begin{aligned}
(x, y, b)_{1} & \mapsto\left(\beta x^{\theta}, \beta y^{\theta}, b^{2 \theta}\right)_{4} \\
(u, v, a)_{2} & \mapsto\left(\alpha^{-1} u^{2 \theta}, \alpha^{-1} v^{2 \theta}, a^{\theta}\right)_{3} \\
(x, y, b)_{3} & \mapsto\left(\beta x^{\theta}, \beta y^{\theta}, b^{2 \theta}\right)_{2} \\
(u, v, a)_{4} & \mapsto\left(\alpha^{-1} u^{2 \theta}, \alpha^{-1} v^{2 \theta}, a^{\theta}\right)_{1}
\end{aligned}
$$

If we restrict the root groups $U_{1}, U_{3}$ to $\{0\} \times\{0\} \times \mathbb{K}$ and $U_{2}, U_{4}$ to $\{0\} \times\{0\} \times \mathbb{K}^{\prime}$, then we obtain the Suzuki-Tits subquadrangle $\mathrm{W}(\mathbb{K}, \phi)$.

\subsection{Moufang sets from exceptional quadrangles of type $\mathbf{F}_{4}$}

The absolute flags of a polarity of a Moufang quadrangle always form a Moufang set. So in the case that an exceptional quadrangle of type $F_{4}$ has a polarity, we also have a Moufang set named $\mathcal{M}(\mathbb{L}, \mathbb{K}, \alpha, \phi)$. We name the flag $\{(\infty),[\infty]\}$ of the quadrangle the element $(\infty)$ of the Moufang set, and the flag $\{((0,0,0),(0,0,0),(0,0,0)),[(0,0,0),(0,0,0),(0,0,0)]\}$ will be denoted as $[(0,0,0),(0,0,0)]$.

The form of a generic element of the root group $U_{\infty}$ of the element $(\infty)$ is 




\section{$1 \frac{1}{1}$

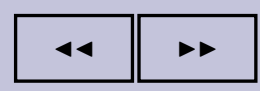 \\ Lemma 4.2. If condition $[\mathcal{T}]$ holds then $\Omega_{(\infty)}^{\prime}$ will be a net.}

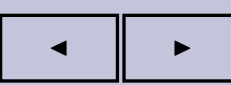

page $8 / 12$

go back

full screen

close

quit

Proof. By condition $[\mathcal{T}]$ one can always choose one line to be a vertical line when checking these properties. We first compute in how many points two such lines will intersect:

- Two vertical lines will never intersect as they form differents orbits of $\left[U_{\infty}, U_{\infty}\right]$;

- One vertical line and one non-vertical line: by using transitivity of $U_{\infty}$ on the non-vertical lines, we can suppose that the non-vertical line is $B_{[(0,0,0),(0,0,0)]}$. The intersection point with a generic vertical block $B_{(x, y, a)}$ is $[(x, y, a),(0,0,0)]$, so these always intersect in exactly one point.

This also proves that the vertical lines form a parallel class for which the second axiom of nets holds, by transitivity we obtain that $\Omega_{(\infty)}^{\prime}$ is a net.

We remark that under assumption of $[\mathcal{T}]$, the parallel class of $B_{[(k, l, m),(u, v, b)]}$ is given by all blocks of the form $B_{\left[(k, l, m),\left(u^{\prime}, v^{\prime}, b^{\prime}\right)\right]}$ (this is easily verified as the exact form in the appendix implies that they do not intersect if $(u, v, b) \neq$ $\left.\left(u^{\prime}, v^{\prime}, b^{\prime}\right)\right)$.

The non-identity elements of $\left[U_{\infty}, U_{\infty}\right]$ will fix $(\infty)$ and all the vertical lines of $\Omega_{(\infty)}^{\prime}$ and acts freely on the points on these lines. If $[\mathcal{T}]$ holds then there would be similar automorphisms of $\Omega$ for other parallel classes than the vertical lines. The following lemma excludes this possibility.

Lemma 4.3. If $\Omega_{(\infty)}^{\prime}$ is a net, then no automorphism of $\Omega$ fixes the point $(\infty)$ and all lines of $\Omega_{(\infty)}^{\prime}$ parallel with $B_{[(0,0,0),(0,0,0)]}$, acts freely on the points of these lines and maps $[(0,0,0),(0,0,0)]$ to $[(0,0,1),(0,0,0)]$.

Proof. Suppose it does exists and let $\tau$ be such an automorphism.

If a line $L$ of $\Omega_{(\infty)}^{\prime}$ would be mapped to a line $L^{\tau}$ of another parallel class then the intersection point $L \cap L^{\tau}$ would be fixed because all the lines parallel to $B_{[(0,0,0),(0,0,0)]}$ are fixed. But we want $\tau$ to act freely on the points of these lines so we have that all parallel classes are stabilized.

Because parallel classes are stabilized we have that $B_{(0,0,0)}^{\tau}=B_{(0,0,1)}$. As a consequence of this we have that the point $[(0,0,0),(u, v, b)]$ will be mapped to $[(0,0,1),(u, v, b)]$ (as both points have to be on the same block $B_{[(0,0,0),(u, v, b)]}$ parallel to $B_{[(0,0,0),(0,0,0)]}$ ). This implies that the block $B_{[(0,0,1),(u, v, b+1)]}$ through the point $[(0,0,0),(u, v, b)]$ will be mapped to the block $B_{[(0,0,1),(u, v, b)]}$. Considering the intersections of these two lines with the fixed line $B_{[(0,0,0),(0,0,0)]}$, we 
have that

$$
\left[\left(\alpha^{-1} u^{2 \theta}, \alpha^{-1} v^{2 \theta}, b^{\theta}\right),(0,0,0)\right]^{\tau}=\left[\left(\alpha^{-1} u^{2 \theta}, \alpha^{-1} v^{2 \theta}, b^{\theta}+1\right),(0,0,0)\right],
$$

or if we rename the variables:

$$
[(x, y, a),(0,0,0)]^{\tau}=[(x, y, a+1),(0,0,0)] .
$$

The generic point $[(x, y, a),(u, v, b)]$ lies on the lines $B_{[(0,0,0),(u, v, b)]}$ and $B_{(x, y, a)}$, these lines are mapped to $B_{[(0,0,0),(u, v, b)]}$ and $B_{(x, y, a+1)}$, so we have that

$$
[(x, y, a),(u, v, b)]^{\tau}=[(x, y, a+1),(u, v, b)] .
$$

If we first apply the automorphism $[(0,0,1),(0,0,0)]_{(\infty)}$ followed by $\tau$, we obtain an automorphism $\tau^{\prime}$ which maps the point $[(x, y, a),(u, v, b)]$ to the point $\left[(x, y, a),\left(u+\beta x^{\theta}, v+x^{\theta}, b+a^{2 \theta}\right)\right]$.

All that is left to show is that the image under $\tau^{\prime}$ of the sphere $S$ with gnarl $[(0,0,0),(0,0,0)]$ through $[(0,0,1),(0,0,0)]$ isn't a sphere, giving us a contradiction. The sphere $S$ will contain the circle $C$ with gnarl $[(0,0,0),(0,0,0)]$ through $[(0,0,1),(0,0,0)]$, which in turn contains the point $[(0,0,1),(0,0,1)]$ (see Appendix B). Hence $S^{\tau^{\prime}}$ will contain the points $[(0,0,0),(0,0,0)],[(0,0,1),(0,0,0)]$ and $[(0,0,0),(0,0,1)]$. As $\Omega_{(\infty)}^{\prime}$ is a net two different spheres of $\Omega^{\prime}$ will have at most two points in common. This implies $S^{\tau^{\prime}}$ will be the sphere through $[(0,0,1),(0,0,0)]$ with gnarl $[(0,0,0),(0,0,1)]$ (because these three points points lay on the circle containing $[(0,0,1),(0,0,0)]$ with gnarl $[(0,0,0),(0,0,1)]$, which is contained in the sphere). Moreover, $\tau^{\prime}$ stabilizes the set $\{[(0,0, a),(0,0, b)] \mid$ $\left.a \in \mathbb{K}, b \in \mathbb{K}^{\prime}\right\}$ so that the circle $C$ will be mapped to the circle with gnarl $[(0,0,1),(0,0,0)]$ through $[(0,0,0),(0,0,1)]$. That this is impossible is shown by Hendrik Van Maldeghem in [8, Lemma 4].

All this has proved that the condition $[\mathcal{T}]$ is false, implying that the gnarls of the spheres are uniquely defined, and that the gnarl of a given sphere can be recognized solely by looking at the properties of the geometry $\Omega^{\prime}$. The last step of our proof is to show that we can reconstruct the exceptional Moufang quadrangle of type $F_{4}$ out of $\Omega^{\prime}$ and see that the automorphisms of $\Omega^{\prime}$ lift to the desired automorphisms of the quadrangle. The construction of the quadrangle goes as follows:

- Points:

- points $x$ of $\Omega^{\prime}$, notation as point of the quadrangle: $x_{p}$,

- spheres $B$ of $\Omega^{\prime}$, notation as point of the quadrangle: $B_{p}$. 



\section{Appendices}

\section{A. Coordinates of points of certain spheres}

page $11 / 12$

go back

full screen

close

quit

$\star$ Spheres going through $(\infty)$ :

- spheres with gnarl $(\infty)$ going through the point $[(x, y, a),(u, v, b)]$ :

$$
B_{(x, y, a)}=\{(\infty)\} \cup\left\{[(x, y, a),(k, l, m)] \mid k, l \in \mathbb{L}, m \in \mathbb{K}^{\prime}\right\}
$$

- spheres with gnarl $[(x, y, a),(u, v, b)]$ (denoted by $\left.B_{[(x, y, a),(u, v, b)]}\right)$ :

$$
\begin{aligned}
B_{[(0,0,0),(u, v, b)]}= & \{(\infty)\} \cup\left\{[(k, l, m),(u, v, b)] \mid k, l \in \mathbb{L}^{\prime}, m \in \mathbb{K}\right\} \\
B_{[(0,0,1),(u, v, b)]}= & \{(\infty)\} \cup\{[(k, l, m+1), \\
& \left.\left.\left(u+\beta k^{\theta}, v+\beta l^{\theta}, b+m^{2 \theta}\right)\right] \mid k, l \in \mathbb{L}^{\prime}, m \in \mathbb{K}\right\}
\end{aligned}
$$

\section{B. Coordinates of points of certain circles}

$\star$ Circles through $(\infty)$ :

- circles with gnarl $(\infty)$ going through the point $[(x, y, a),(u, v, b)]$ :

$$
\{(\infty)\} \cup\left\{[(x, y, a),(u, v, k)] \mid k \in \mathbb{K}^{\prime}\right\}
$$

- circles with gnarl $[(x, y, a),(u, v, b)]$ :

$$
\{(\infty)\} \cup\left\{\left[(x, y, a+k),\left(u, v, b+a^{2} k^{2 \theta}+k^{2 \theta} \alpha\left(x \bar{x}+\beta^{2} y \bar{y}\right)\right)\right] \mid k \in \mathbb{K}^{\prime}\right\}
$$

$\star$ The circle with gnarl $[(0,0,0),(0,0,0)]$ through $[(0,0,1),(0,0,0)]$ :

$$
\begin{aligned}
& \{[(0,0,0),(0,0,0)]\} \\
& \cup\left\{\left[\left(0,0, \frac{x}{1+x+x^{2 \theta}}\right),\left(0,0, \frac{1}{1+x^{2 \theta}+x^{2}}\right)\right] \mid x \in \mathbb{K}\right\}
\end{aligned}
$$

$\star$ The circle through $[(0,0,1),(0,0,0)]$ with gnarl $[(0,0,0),(0,0,1)]$ :

$$
\begin{aligned}
& \{[(0,0,0),(0,0,0)]\} \\
& \cup\left\{\left[\left(0,0, \frac{1}{1+x+x^{2+2 \theta}}\right),\left(0,0, \frac{x^{2 \theta}}{1+x^{2 \theta}+x^{2+4 \theta}}\right)\right] \mid x \in \mathbb{K}\right\}
\end{aligned}
$$




\section{References}

[1] F. Haot, K. Struyve and H. Van Maldeghem, Ree geometries, Forum Math., to appear.

[2] B. Mühlherr, Locally split and locally finite twin buildings of 2-spherical type, J. Reine Angew. Math. 511 (1999), 119-143.

[3] B. Mühlherr and H. Van Maldeghem, Exceptional Moufang quadrangles of type $\mathrm{F}_{4}$, Canad. J. Math. 51 (1999), 347-371.

[4] _ Moufang sets from groups of mixed type, J. Algebra 300 (2006), 820-833.

[5] M. Ronan and J. Tits, Building buildings, Math. Ann. 278, 291-306.

[6] J. Tits and R. Weiss, Moufang Polygons, Springer Monogr. Math., SpringerVerlag, 2002.

[7] H. Van Maldeghem, Generalized Polygons, Monogr. Math. 93, Birkhäuser Verlag, 1998.

[8] _ Moufang lines defined by (generalized) Suzuki groups, European J. Combin. 28 (2007), 1878-1889.

Koen Struyve

Department of Pure Mathematics and Computer Algebra, Ghent University, KrijgslaAn 281 - S22, B-9000 GENT, BELGIUM

e-mail: kstruyve@cage.ugent.be 